\title{
RECURSOS DIGITAIS PARA O ENSINO E APRENDIZAGEM DE INGLÊS
}

\author{
Celso Henrique Soufen TUMOLO \\ Universidade Federal de Santa Catarina \\ Caroline Chioquetta LORENSET \\ Instituto Federal de Santa Catarina \\ Nayara Nunes SALBEGO \\ Instituto Federal de Santa Catarina
}

\begin{abstract}
RESUMO: Recursos tecnológicos podem auxiliar o processo de ensino e aprendizagem como um todo e, em particular, de língua estrangeira. Este texto busca trazer uma reflexão sobre recursos digitais como auxiliares para o ensino e aprendizagem de língua estrangeira, focando em três recursos, a saber: histórias digitais, videogames e webconferência. Tais recursos podem ser utilizados dentro ou fora de sala de aula. Assim, os objetivos deste texto são: (a) caracterizar cada recurso digital, abordando referencial teórico na área; e (b) trazer uma reflexão sobre como cada recurso digital pode ser usado para ensino e aprendizagem de língua estrangeira. As discussões são apresentadas a fim de permitir o estabelecimento de bases para o trabalho pedagógico de professores de línguas estrangeiras. Assim, com a apresentação dos recursos digitais e reflexão sobre o uso de cada um na área, espera-se contribuir para a discussão sobre o uso de recursos digitais para o ensino e aprendizagem de línguas estrangeiras, como também para a incorporação desses recursos para a prática docente de professores na área de ensino e aprendizagem de Inglês como língua estrangeira.
\end{abstract}

PALAVRAS-CHAVE: tecnologia educacional; recursos digitais; ensino e aprendizagem de língua

ABSTRACT: Digital resources can assist the teaching and learning process as a whole and, in particular, a foreign language. This text aims to bring a reflection on digital resources as tools to the teaching and learning of foreign languages, focusing on three resources, namely: digital stories, videogames and webconferencing. Such resources can be used in or outside the classroom. Thus, the objectives of this text are: (a) to characterize each digital resource, addressing the theoretical bases in the area; and (b) bring a reflection on how each digital resource can be used for foreign language teaching and learning. The discussions are presented in order to establish the basis for the pedagogical work of foreign language teachers. Thus, the presentation of the digital resources and of a reflection on the use of each one in the area is expected to contribute to the debate on the use of digital resources as well as to the incorporation of these resources in the teachers' practice in the area of teaching and learning English as a foreign language.

KEYWORDS: educational technology; digital resources; second language instruction

\section{Introdução}

Recursos tecnológicos podem auxiliar o processo de ensino e aprendizagem como um todo e, em particular, de língua estrangeira. A presença ubíqua dos mais variados aparatos tecnológicos facilita acesso à informação e, consequentemente, tornam oportunidades de aprendizagem mais acessíveis (CHAPELLE, 2003; BLAKE, 2005). No entanto, não basta se conectar à rede; é preciso reflexão e planejamento sobre quais recursos utilizar, principalmente de forma pedagógica (MIKI; BRIAN; YUSUKE, 2009; THOMAS; 
REINDERS; WARSCHAUER, 2014). Neste sentido, o objetivo deste artigo é tratar da utilização de recursos digitais que podem propiciar oportunidades de ensino e aprendizagem, especificamente na área de línguas estrangeiras.

Tumolo (2014) traz uma reflexão sobre vários recursos digitais como auxiliares para o ensino e aprendizagem de línguas estrangeiras. Considerando a relevância do tema, este texto busca estender a reflexão para o uso de histórias digitais, videogames e webconferência. Tais recursos podem ser utilizados dentro ou fora de sala de aula. Assim, os objetivos deste texto são: (a) caracterizar cada recurso digital, abordando referencial teórico na área; e (b) trazer uma reflexão sobre como cada um pode ser usado para ensino e aprendizagem de língua estrangeira. As discussões são apresentadas a fim de permitir o estabelecimento de bases para o trabalho pedagógico e prática docente de professores de línguas estrangeiras. O texto está organizado com uma descrição breve de cada recurso, seguida de aporte teórico justificando seu uso e sugestões para incorporação na prática docente do professor.

\section{Histórias Digitais e Ensino e Aprendizagem de Línguas Estrangeiras}

Histórias Digitais (doravante HDs) são recursos digitais multimidiáticos e multimodais, sendo caracterizados, grosso modo, de acordo com Tumolo (2015), como um vídeo produzido resultante da combinação de imagens digitais, textos, vídeos, música de fundo e de efeitos especiais, e principalmente uma narração oral gravada, com tempo de duração entre 2 a 10 minutos, com histórias sobre temas diversos, sempre narradas a partir da perspectiva de seu autor.

Como composição multimidiática e multimodal, HDs combinam imagens e outros meios, com a possibilidade de que a informação desejada seja compreendida pela combinação de textos (orais e/ou escritos) e imagens. Em sua teoria cognitiva da aprendizagem multimídia, Mayer (2009) aponta pressupostos justificando a adoção dessas composições, dentre eles, a o do canal duplo, em que humanos fazem uso de informações distintas para representações visuais e para representações verbais. $O$ autor enfatiza que, com uma explicação multimidiática, com palavras e imagens, a compreensão da informação pode ser aumentada e, com isso, resultar em uma maior probabilidade de aprendizagem. Assim, o autor afirma que composições multimidiáticas promovem o aprendizado mais profundo, permitindo o estabelecimento de conexões entre imagens e palavras e a integração com o conhecimento prévio.

O autor, buscando dar consistência à sua proposição, faz referências à Teoria da Dupla Codificação, de Allan Paivio, que propõe que as imagens e as palavras são processadas de formas diferentes, por dois diferentes canais - verbal ou visual, e armazenadas na memória visual e na memória verbal, com a possibilidade de interação entre os canais, o que fortalece a memorização devido às associações possíveis pela dupla codificação, e criação de redundância na mensagem multimodal.

HDs podem ser usadas para fins diversos e, em educação, essencialmente como elemento que evoca grande motivação. Podem ser usadas para ensino e aprendizagem de língua estrangeira, também, dentro dos princípios da abordagem por conteúdo como também dentro dos princípios da abordagem por projetos.

A abordagem por conteúdo permite exposição de aprendizes ao insumo linguístico e uso autêntico da língua alvo. Ela implica uma integração da aprendizagem de língua e 
aprendizagem de conteúdo, e a proficiência linguística é alcançada com foco em estudo de conteúdos disciplinares por meio da língua (STRYKER; LEAVER, 1997).

Esta abordagem permite, também, exposição à língua ao aprender sobre um conteúdo, oportunidades do uso de conhecimento prévio sobre o conteúdo e sobre a língua, envolvimento de várias formas de aprendizagem, como a cooperativa, e inclusão de conteúdos de interesses dos aprendizes (GRABE; STOLLER, 1997).

O uso de HDs, nesta abordagem, para aprendizagem de LE resulta, naturalmente, na integração das quatro habilidades linguísticas - escrita, leitura, fala e audição, pois os aprendizes de LE são encorajados a pensar e aprender por meio do uso da língua-alvo integrando essas habilidades (MADRID; GARCIA SÁNCHEZ, 2001).

A abordagem por projetos permite o desenvolvimento de projetos, envolvendo aprendizes na elaboração e desenvolvimento de seus projetos, orientados por um professor. Neste caso, há uma mudança dos papéis do professor, que deixa de ser o especialista no conteúdo, e do aluno, que passa a seguir suas próprias questões, criando seus próprios significados (BOSS; KRAUSS, 2007).

Essa abordagem permite, também, o uso de conteúdo significativo, das competências do século 21, como solução de problemas, pensamento crítico, colaboração, comunicação e criatividade/inovação, de investigação profunda, com engajamento em perguntar e respostas, de questão motivadora, considerada intrigante, de voz e escolha, com escolhas sobre o produto a ser criado e o método de trabalho, de crítica e revisão, com oferecimento e recebimento de feedback sobre a qualidade do projeto, para revisões e mais investigações (TUMOLO, 2015).

Como projeto, o trabalho colaborativo pode fazer da LE alvo a língua de comunicação e negociação de significados, em que há a necessidade de comunicação, em particular com o uso de feedback de pares em todas as fases de elaboração de uma HD, feedback este que pode ser forma escrita ou oral, envolvendo as quatro habilidades de forma integrada.

O uso de HDs, nesta abordagem, para aprendizagem de LE permite atingir objetivo significativo em sala de aula, pois aprendizes praticaram a LE envolvidos em comunicação real e autêntica, criando HDs envolventes e profundas na LE (CASTAÑEDA, 2013). Também, as atividades envolvidas no processo de criação de HDs podem permitir oportunidades de prática de fluência e interação significativa, como também de prática de melhoramento da pronúncia (LANNOTTI, 2004).

Assim, dentro desses princípios dessas abordagens, HDs como recursos digitais, podem auxiliar no desenvolvimento de uma língua estrangeira.

\section{Jogos Digitais e Ensino e Aprendizagem de Línguas Estrangeiras}

Como recurso digital para ensino e aprendizagem de línguas estrangeiras, jogos digitais são considerados ambientes interativos, de fácil motivação e com rápido feedback ao jogador, oferecendo opções de customização para cada jogador (PRENSKY, 2001; GEE, 2005). De acordo com Prensky (2005), jogos digitais são caracterizados por seis elementos principais, que formam a estrutura básica dos bons jogos, a saber: objetivos, feedback, conflito, competição, desafio, interação e narrativa. Além destes elementos básicos de 
estrutura, há os gêneros dos jogos, que determinam o tom das missões e objetivos dos jogos, podendo se entrelaçar e se misturar em um mesmo jogo. Eles são oito gêneros: ação, aventura, interpretação, luta, estratégia, simulação, esportes, e enigmas. A título de exemplo, no jogo que será analisado neste artigo, The Sims, o gênero que se sobressai é o de simulação.

Além dos elementos estruturais e dos gêneros, jogos digitais ainda podem ser divididos em jogos complexos e minijogos. Prensky (2005) explica a diferença entre eles conforme tabela abaixo:

\begin{tabular}{|c|c|}
\hline Jogos Complexos & Minijogos \\
\hline Grande complexidade & Pouca complexidade \\
\hline $\begin{array}{l}\text { Requerem habilidades, identidade virtual, } \\
\text { objetivos, interação, dilemas e escolhas }\end{array}$ & Para relaxamento ou exercício do cérebro \\
\hline Exigem concentração e atenção do jogador & Exigem tarefas específicas \\
\hline $\begin{array}{l}\text { Contém uma mistura de desafios de } \\
\text { dificuldade crescente que se entrelaçam }\end{array}$ & Lidam com apenas um assunto ou tarefa \\
\hline Jogadores múltiplos & Jogador único \\
\hline $\begin{array}{l}\text { São vendidos em lojas de jogo ou versões } \\
\text { mais básicas podem ser baixadas }\end{array}$ & Podem ser baixados gratuitamente \\
\hline
\end{tabular}

Tabela 1. Elementos estruturais dos jogos complexos e minijogos.

Assim, de acordo com Prensky (2005), jogos complexos requerem maior habilidade dos jogadores, além de uma nova identidade virtual, que pode ou não ser similar à da vida real do jogador, permitindo interação tanto com o jogo, quanto com outros jogadores. Por conseguinte, eles exigem atenção constante do jogador e crescente concentração para cumprir desafios de dificuldade gradativa. Civilization III, EverQuest e World of Warcraft são exemplos de jogos complexos, que permitem jogadores múltiplos ${ }^{1}$. Em contrapartida, os minijogos têm pouca complexidade e, exatamente por isso, são jogados para fins de relaxamento, lazer, ou exercício do cérebro. Estes jogos ${ }^{2}$ exigem tarefas específicas de jogadores únicos, lidando com apenas um assunto ou tarefa. Exemplos de minijogos são Candy Crush, Bejewled, Scrabble e Paciência.

Godwin-Jones (2014) afirma que os jogos digitais permitem o aprendizado de línguas estrangeiras por constituírem-se de ambientes interativos e imersivos, onde a língua alvo é usada repetidamente no jogo, oportunizando o aprendizado em contexto e coerentemente. Porém, para o autor, para que o jogo possa oferecer benefícios múltiplos, deve-se observar a abordagem ou método de ensino utilizados para incluir o jogo no contexto de aprendizado. Atividades realizadas antes, durante e depois das sessões de jogos são importantes para que o aprendizado da língua aconteça de forma contextualizada e contínua. Além de inúmeras vantagens que podem ser observadas ao adaptar jogos digitais de entretenimento para objetivos educacionais, Godwin-Jones (2014) assegura ainda que há vantagens como o letramento digital e oportunidades para que cada jogador estabeleça suas preferências e interesses pessoais em cada jogo.

A inserção de jogos digitais no contexto escolar pode promover interação, motivação e agência nos alunos (REIS; BILIÃO, 2014; MILLER; HEGELHEIMER, 2006), pois jogos digitais ensinam, inspiram, envolvem e unem seus usuários, apresentando grande potencial

\footnotetext{
1 Jogos complexos podem ser baixados em sua versão mais simples online, apesar de suas versões mais completas estarem disponíveis somente se compradas.

${ }^{2}$ Minijogos podem ser baixados em computadores, tablets ou smartphones de forma gratuita.
} 
para atender às necessidades educacionais. Ranalli (2008) afirma que existem vários benefícios com jogos digitais em sala de aula (notadamente os jogos de simulação), incluindo a promoção do processamento cognitivo e do envolvimento ativo, além do uso da língua estrangeira como um meio de comunicação. Jogos de simulação, com narrativas similares à da vida real, ademais, unem conhecimentos específicos de jogo, oferecendo oportunidades de uso da língua em contexto.

The Sims é um jogo digital de simulação muito pesquisado academicamente como recurso digital para ensino e aprendizagem de línguas estrangeiras, pois permite que jogadores escolham dentre diversas opções de idiomas para jogar. Consequentemente, jogadores se sentem motivados em função da sua narrativa ser muito próxima à da vida real, incluindo tarefas e objetivos que giram em torno de vida social, vida acadêmica e profissional, além de alimentação, higiene e lazer. Muitos estudos (MENEZES; SCHLEMMER, 2014; MENEZES, 2013; YUDINTSEVA, 2015) afirmam que este jogo é divertido e cativante em função do seu envolvimento no contexto da vida real que a narrativa traz, ao oferecer, ao jogador, opções de local de trabalho, personalidade e características físicas, por exemplo, ele promove o uso da língua-alvo, especialmente o vocabulário, oportunizando para que isto aconteça com coerência e em contexto, e representa uma forma interessante para ampliar e contextualizar a aprendizagem de inglês como língua estrangeira.

Ranalli (2008) aponta que o jogo de simulação The Sims contribui de fato para a aquisição de língua estrangeira, e participantes de sua pesquisa afirmaram sentir que o jogo tem potencial de contribuir para a aprendizagem de línguas. Para Purushotma (2005), The Sims é um bom exemplo de jogo de entretenimento com alto valor educacional, rico em associações (palavras e texto escrito associados com imagens, ações, animações), tornando a aprendizagem automática e natural, e criando oportunidades de repetição contínua em ricos contextos para aprendizado da língua estrangeira. Neste sentido, com o uso de imagens e as palavras (orais ou escritas), a memorização pode ser fortalecida, devido às associações possíveis pela dupla codificação oriunda dos canais verbal e visual (MAYER, 2009).

Durante o jogo, o stress dos jogadores diminui, propiciando aos jogadores um estado de prazer e relaxamento, onde a aprendizagem incidental acontece. De acordo com Noroozloo (2015), a aprendizagem incidental é facilitada ao jogar The Sims pois os participantes aprendem novas palavras brincando ao interagir com cenas e personagens simulados. A título de exemplo, uma das variáveis que o jogador deve prestar atenção em The Sims é o nível de energia dos personagens. Se um jogador tentar adivinhar o significado da palavra de forma incorreta, o personagem vai tomar algumas atitudes para avisar ao jogador sua vontade e/ou necessidade, como se tornar sonolento e pensar muito em camas, em noite ou em dormir. Porém, caso o jogador ainda não reconheça a ação a ser tomada, o personagem cairá em sono profundo onde ele estiver, podendo ser em casa, no supermercado ou no jardim. De acordo com o autor, estas ações beneficiam o aprendizado incidental, criando no jogador o interesse nas ações que devem ser realizadas para cumprir as missões.

Para concluir, muitos estudos (PRENSKY, 2001; GEE, 2005; SQUIRE, 2006, CHIK, 2014; YUDINTSEVA, 2015) na área de ensino e aprendizagem de línguas indicam que jogos digitais auxiliam muitos aprendizes de inglês como língua estrangeira, desenvolvendo tanto conhecimentos específicos quanto diversos, auxiliando na aquisição de vocabulário, na comunicação e interação entre jogadores nativos e não-nativos falantes de inglês, além de fornecer autonomia, auxiliando no desenvolvimento cognitivo e social como um todo. Jogos digitais, além disso, podem fornecer estratégias muito efetivas para o desenvolvimento da aprendizagem, como o uso das repetições e aprendizagem contextualizada, motivando jogadores a continuar no jogo para cumprir missões e passar de níveis. 


\section{Webconferência e Ensino e Aprendizagem de Línguas Estrangeiras}

O recurso digital webconferência possibilita comunicação oral à distância, fazendo com que pessoas distantes fisicamente possam se comunicar oralmente através do uso de computador, tablet, celular, desde que tenham um microfone. É possível utilizar a webcam, caso os interagentes queiram se ver virtualmente, ou é possível apenas utilizar o canal de voz. Além disso, existe a possibilidade de chat, envio de arquivos e compartilhamento de tela, além de outros recursos, dependendo do software ou aplicativo utilizado.

Recursos de webconferência podem ser utilizados para fins didáticos, pois recriam à distância condições semelhantes àquelas de um encontro presencial. Além disso, o recurso promove a possibilidade de se atingir um número maior de aprendizes, distantes fisicamente, proporcionando oportunidade de interação na língua-alvo. Cruz e Barcia (2000) definem webconferência como uma tecnologia que permite a comunicação face a face de grupos distantes, através de sinais em áudio e vídeo. Dentre os mais populares, por serem gratuitos, estão o Skype e o Google Hangouts.

O Skype é um recurso gratuito e popular de webconferência, o qual pode ser usado para fins didáticos, através do qual até 25 pessoas podem se conectar em uma única chamada. A possibilidade de aprender pelo $S k y p e^{3}$ beneficia alunos que não têm acesso a aulas em contextos mais tradicionais, como a sala de aula, ou aqueles que têm problemas com tempo ou deslocamento, dentre outras situações.

Dentre os softwares para webconferência, o Skype se destaca por ser de fácil acesso e gratuito. Existe a versão para computador e celular, facilitando ainda mais o download e uso. Salbego e Tumolo (2015) realizaram uma pesquisa que investiga percepções de alunos que tiveram experiências de aulas presenciais e online por Skype. A percepção dos participantes apontou aspectos positivos, enfatizando o desenvolvimento de fala e escuta através do uso do Skype para fins didáticos. Os participantes afirmaram que são necessários a atenção, para entender a conversa entre os interagentes e também o esforço, para se expressar verbalmente e se fazer entender. Tais características são propícias para a aprendizagem de línguas, pois fomentam a produção oral e a escuta.

No entanto, o software não foi desenvolvido para fins didáticos; o Skype não apresenta um quadro branco como o Flashmeeting, o qual imita o quadro branco de uma sala de aula regular (HAMPEL; STICKLER, 2012), mas apresenta uma caixa de chat que pode substituir tal recurso. A diferença é que no quadro branco apenas o professor escreve conteúdos relativos à aula, enquanto que na caixa de texto do Skype, as conversas paralelas podem acontecer concomitantes às anotações da aula.

Outra diferença é que no Skype não é possível que os alunos 'levantem a mão', como no Flashmeeting, para sinalizar que querem se expressar, apenas clicando em um botão que organiza os turnos de fala (HAMPEL; STICKLER, 2012). Nesse sentido, ele pode ser mais efetivo para aulas com poucos alunos, a fim de que as tomadas de turno não se interponham.

\footnotetext{
${ }^{3}$ Desde o seu lançamento em 2003, o Skype é uma ferramenta popular e muitas escolas de idiomas adotam tal recurso para suas aulas. Exemplos de escolas de idiomas que utilizam o recurso digital Skype para as aulas: http://www.easyespanol.org/; http://nativeenglishteacher.net/; http://skype-englishschool.com/.
} 
Há ainda pouca pesquisa investigando o recurso de webconferência para aulas de línguas (SANTOS, 2012; VERJANO 2013; GUO 2013), mas os resultados apontam aspectos positivos com relação à aprendizagem e à interação entre os alunos. No Brasil, Santos (2012) analisou o uso de Skype para o desenvolvimento das quatro habilidades em Inglês e os resultados apontaram que as habilidades mais desenvolvidas foram a audição (listening) e a fala (speaking), similar ao estudo de Salbego e Tumolo (2015).

Verjano (2013) investigou a interação online pelo Skype, contrastando com a interação presencial. A autora identificou produção linguística mais variada, além de maior número de alunos interagindo nas aulas online. Guo (2013), por sua vez, realizou um estudo com aprendizes chineses para avaliar suas preferências com relação ao meio multimodal de aprendizagem. $\mathrm{O}$ autor concluiu que alunos adolescentes tendem a usar a língua materna nas aulas, enquanto que nos encontros por webconferência eles se esforçaram para utilizar a língua-alvo, nesse caso o Inglês. Dessa forma, o meio online propiciou a produção na língua estrangeira.

Assim, embora haja ainda a necessidade de se entender de forma concreta como a aprendizagem acontece em interações online por webconferência, que focam em aulas de língua através de recursos de webconferência e em possibilidades didáticas para professores que trabalham ou almejam trabalhar com esta modalidade de ensino para o desenvolvimento de língua, especialmente de interação oral, é possível afirmar que o recurso de webconferência tem potencial para auxiliar o ensino e a aprendizagem de língua estrangeira.

\section{Considerações Finais}

Recursos digitais têm grande potencial para auxiliar processos educativos, em particular o processo de ensino e aprendizagem de uma língua estrangeira. Este texto trouxe uma reflexão sobre três recursos, historias digitais, jogos digitais e webconferência.

HDs podem ser usadas apenas como elemento motivador, ou mesmo para exposição ao insumo linguístico na língua-alvo. Porém, elas podem ter um papel mais fundamental nas abordagens por conteúdo e/ou por projetos, sendo acomodadas dentro delas, quer seja apenas com a adoção de alguns de seus princípios ou com a adoção das abordagens como um todo e, assim, contribuindo para o desenvolvimento de uma língua estrangeira.

Jogos digitais como recurso para ensino e aprendizagem trazem inúmeras vantagens ao jogador/aprendiz, como autonomia, motivação e interesse para continuar jogando e aprendendo de forma incidental. Jogos de simulação, em especial, por assemelharem-se às narrativas da vida real (incluindo vocabulário de higiene, alimentação e vida social, por exemplo), oportunizam o aprendizado contextualizado e com coerência de uma língua estrangeira.

O recurso digital webconferência possibilita a interação oral de aprendizes de línguas, oportunizando a comunicação entre indivíduos fisicamente distantes. O tipo de interação fomentado se assemelha ao de situações reais por ser um meio síncrono de comunicação. Dessa forma, o recurso pode contribuir para o desenvolvimento de uma língua estrangeira.

Com os recursos digitais apresentados, ampliam-se as oportunidades de aprendizagem de línguas. Professores e alunos em diferentes contextos podem usufruir de tais recursos para 
diversificar atividades rotineiras, motivando e fazendo uso de uma realidade presente no seu dia a dia.

\section{Referências}

BLAKE, R. Bimodal CMC: The glue of language learning at a distance. CALICO, v. 22, n. 3, p. 497-511, 2005.

BOSS, S.; KRAUSS, J.; CONERY, L. Reinventing Project-Based Learning: your field guide to real-world projects in the digital age. Washington, D. C.: International Society for Technology in Education, 2007.

CASTAÑEDA, M. "I am proud that I did it and it's a piece of me": digital Storytelling in the foreign language classroom. CALICO Journal, 30, v. 1, p. 42-62, 2013.

CHAPELLE, C. English Language Learning and Technology. John Benjamins Publishing, 2003.

CHIK, A. "Digital gaming and language learning: Autonomy and community". In: Language Learning \& Technology, v. 18, n. 2, p. 85-100, 2014.

CRUZ, D. M.; BARCIA, R. M. Educação a distância por videoconferência.

Tecnologia Educacional. Rio de Janeiro, ano XXVIII, v. 29, n. 150/151, p. 3-10, jul./dez. 2000.

CRUZ, D. M.; MORAES, M. Manual de Videoconferência. Florianópolis: Laboratório de Ensino a Distância: UFSC, 1998.

GEE, J. P. Why video games are good for your soul: pleasure and learning. Melbourne: Common Ground, 2005.

GODWIN-JONES, R. Games in language learning: Opportunities and challenges. Language Learning \& Technology, v. 18, n. 2, p. 9-19. 2014.

GRABE, W.; STOLLER, F. L. Content-based Instruction: research foundations. In: SNOW, M. A.; BRINTON, D. The content-based classroom: perspectives on integrating language and content. NY: Longman, 1997, p. 5-21.

GUO, S. Applying web-conferencing in a beginnerrs' chinese class. 30th ASCILITE Conference 2013 Proceedings. Macquire University, Sydney, Austrália, 2013.

HAMPEL, R.; STICKLER, U. The use of videoconferencing to support multimodal interaction in an online classroom. ReCALL, v. 24, n. 2, 116-137, 2012.

LANNOTTI, E. How to make crab soup: digital storytelling projects for ESL students. Transit, $\quad$ p. 10-12, 2004. Disponível em: $<$ http://ctl.laguardia.edu/journal/pdf/InTransit_v1n1_DigitalStorytellingESL.pdf $>$ Acesso em: 12 de maio de 2014. 
MADRID, D.; GARCIA SÁNCHEZ, E. Content-based second language teaching. In: GARCIA SÁNCHEZ, E. (ed.): Present and Future Trends in TEFL. Universidad de Almería: Secretariado de publicaciones, 2001, p. 101-134.

MAYER, R. Multimedia learning. 2. ed. Cambridge University Press, NY. 2009.

MENEZES, J. Jogos sociais digitais como ambiente de aprendizagem da língua inglesa. Dissertação de mestrado. Unisinos: São Leopoldo. 2013.

MENEZES, J.; SCHLEMMER, E. Desenvolvendo a competência comunicativa através de Social Games nas aulas de Língua Inglesa. In: Proceedings of SBGames 2014, XIII SBGames - Porto Alegre, RS, Brazil, November $12^{\text {th }}-14^{\text {th }}, 2014$. Pp. 543-549.

MILLER, M.; HEGELHEIMER, V. The SIMs meet ESL: Incorporating authentic computer simulation games into the language classroom. In: Interactive Technology \& Smart Education, v. 4, p. 311-328. 2006.

NOROOZLOO, N. ET AL. The Effect of Using a Digital Computer Game (SIMS) on Children's Incidental English Vocabulary Learning. In: Science Journal (CSJ) Special Issue: The Second National Conference on Applied Research in Science and Technology, v. 36, n. 3, pp. $1991-2000.2015$.

PRENSKY, M. Digital game-based learning. New York: McGraw-Hill. 2001.

PRENSKY, M. In Educational Games, Complexity Matters: Mini-games are Trivial - but "Complex" Games Are Not - An Important Way for Teachers, Parents and Others to Look at Educational Computer and Video Games. In: Educational Technology, v. 45, n. 4, p. 22-28. 2005.

PURUSHOTMA, R. Commentary: You're not studying, you're just... In: Language Learning \& Technology, v. 9, n. 1, p. 80-96. 2005.

RANALLI, J. Learning English with The Sims: exploiting authentic computer simulation games for L2 learning. In: Computer Assisted Language Learning, v. 21, issue 5, p. 441455. 2008.

REIS, S.C. \& Bilião, M. O Uso de Jogos Digitais nas áreas de Educação e Letras em Publicações Brasileiras: Mapeando o Estado da Arte. In: Cenários, Porto Alegre, n.10, $2^{\circ}$ semestre 2014, pp. 4-17. ISSN 2177-1960. 2014.

SALBEGO, N. N.; TUMOLO, C. H. S. Skype ${ }^{\mathrm{TM}}$ classes: teachers and students' perceptions on synchronous online classes in relation to face-to-face teaching and learning. International Journal of Language and Applied Linguistics (IJLAL). Irã, 2015.

SANTOS, D. S. Ensino/Aprendizagem da Língua Inglesa com o auxílio da ferramenta Skype. Dissertação (Monografia em Língua Inglesa e Literaturas) - Universidade Federal da Bahia, Bahia, 2012.

SQUIRE, K. "From Content to Context: Videogames as Designed Experience". In: Educational Researcher, 35, v. 8, p. 19-29. Sage Publications. 2006.

STRYKER, S.; LEAVER, B. L. Content-based instruction in foreign language education: models and methods. Georgetown University Press, 1997. 
TSUKAMOTO, M; NUSPLIGER, B; SENZAKI, Y. Using Skype $\subset$ to connect a classroom to the world: Providing students an authentic language experience within the classroom. CamTESOL Conference on English Language Teaching: Selected Papers, v. 5, p. 162$168,2009$.

TUMOLO, C. H. S. Recursos digitais e aprendizagem de inglês como língua estrangeira. Revista Ilha do Desterro, número 66, pp 203-238. 2014. Disponível em: <http://dx.doi.org/10.5007/2175-8026.2014n66p203>. Acesso em: 03 maio 2015.

TUMOLO, C. H. S. Histórias digitais como recurso para ensino/aprendizagem de inglês como língua estrangeira. Revista Estudos Anglo-Americanos, n. 43, p. 101-117. 2015. Disponível em: <http://reaa.ufsc.br/index.php/reaa/article/view/1322>. Acesso em: 02 abril 2016.

VERJANO, A. C. Using Skype in a Primary Class: a case study. Bellaterra Journal of Teaching and Learning Language and Literature. v. 6, n. 2, p.49-68, maio/jun. 2013.

YUDINTSEVA, A. Game-Enhanced Second Language Vocabulary Acquisition Strategies: A Systematic Review. In: Open Journal of Social Sciences 2015, v. 03, p. 101-109. 2015. 